\title{
Compressive Sensing Based Channel Estimation for Massive MIMO Communication Systems
}

\author{
Athar Waseem (DD, ${ }^{1}$ Aqdas Naveed, ${ }^{1}$ Sardar Ali, ${ }^{2}$ Muhammad Arshad, \\ Haris Anis, ${ }^{1}$ and Ijaz Mansoor Qureshi ${ }^{4}$ \\ ${ }^{1}$ International Islamic University, Islamabad, Pakistan \\ ${ }^{2}$ University of Buner, Pakistan \\ ${ }^{3}$ University of Peshawar, Pakistan \\ ${ }^{4}$ AIR University, Islamabad, Pakistan \\ Correspondence should be addressed to Athar Waseem; athar.waseem@iiu.edu.pk
}

Received 9 November 2018; Revised 13 March 2019; Accepted 21 April 2019; Published 27 May 2019

Guest Editor: Sungchang Lee

Copyright (c) 2019 Athar Waseem et al. This is an open access article distributed under the Creative Commons Attribution License, which permits unrestricted use, distribution, and reproduction in any medium, provided the original work is properly cited.

\begin{abstract}
Massive multiple-input multiple-output (MIMO) is believed to be a key technology to get 1000x data rates in wireless communication systems. Massive MIMO occupies a large number of antennas at the base station (BS) to serve multiple users at the same time. It has appeared as a promising technique to realize high-throughput green wireless communications. Massive MIMO exploits the higher degree of spatial freedom, to extensively improve the capacity and energy efficiency of the system. Thus, massive MIMO systems have been broadly accepted as an important enabling technology for 5th Generation (5G) systems. In massive MIMO systems, a precise acquisition of the channel state information (CSI) is needed for beamforming, signal detection, resource allocation, etc. Yet, having large antennas at the BS, users have to estimate channels linked with hundreds of transmit antennas. Consequently, pilot overhead gets prohibitively high. Hence, realizing the correct channel estimation with the reasonable pilot overhead has become a challenging issue, particularly for frequency division duplex (FDD) in massive MIMO systems. In this paper, by taking advantage of spatial and temporal common sparsity of massive MIMO channels in delay domain, nonorthogonal pilot design and channel estimation schemes are proposed under the frame work of structured compressive sensing (SCS) theory that considerably reduces the pilot overheads for massive MIMO FDD systems. The proposed pilot design is fundamentally different from conventional orthogonal pilot designs based on Nyquist sampling theorem. Finally, simulations have been performed to verify the performance of the proposed schemes. Compared to its conventional counterparts with fewer pilots overhead, the proposed schemes improve the performance of the system.
\end{abstract}

\section{Introduction}

Multiple-input multiple-output (MIMO) systems have multiple antennas at both the transmitter and receiver ends. By addition of multiple antennas, higher degree of freedom in wireless channels (in terms of time and frequency dimensions) can be obtained in order to achieve target of high data rates. For this reason, major performance progress can be attained in terms of system reliability and both spectral and energy efficiency. And also such higher degrees of freedom can be exploited using beamforming given that channel state information is available. There are large number of antenna elements (around tens or even hundreds) deployed at both sides, the transmitter and receiver. It is important to note that the transmit antennas may be distributed or colocated in different applications. Also, the huge number of receive antennas can be acquired by one device or distributed to many devices $[1,2]$. Additionally, massive MIMO systems help in minimizing the effects of noise and fast fading, and also intracell interference can be reduced using straightforward detection and linear precoding methods. By appropriately implementing multiuser MIMO (MU-MIMO) in massive MIMO systems, the design of medium access control (MAC) layer can be more simplified by getting rid of complicated scheduling algorithms [2]. With MU-MIMO, signals to individual users can be easily separated using the 
same time-frequency resource, at base station. Therefore, these main advantages make it feasible to introduce the massive MIMO system as a promising candidate for $5 \mathrm{G}$ wireless communication networks.

One of the major issues in massive MIMO systems is the accurate acquisition of the channel state information (CSI) for beamforming, resource allocation, signal detection, etc. Due to large antennas placed at the BS, the estimation of channels linked with hundreds of transmit antennas is required at users which results in high pilot overhead. Hence, the precise channel estimation with the low pilot overhead is a challenging task $[2,3]$.

There are several research challenges which are required to be solved before massive MIMO can be fully integrated into future wireless systems $[4,5]$ :

(i) Beamforming will involve a huge amount of channel state information, which will be challenging for the downlink.

(ii) MIMO can be unfeasible for FDD systems, but can be employed in time division duplex (TDD) systems due to having channel reciprocity.

(iii) Conventional channel estimation approaches require a large pilot and feedback overhead, which typically scales proportionally with number of BS transmit antennas, which results in unfeasible condition for large-scale FDD MIMO systems.

(iv) In addition, massive MIMO experiences pilot contamination from other cells if the transmit power is high; otherwise it will be affected by thermal noise. Methods will be required to solve this in order to deliver the promised performance.

(v) There is a need for channel models of massive MIMO systems, without which it will be difficult for researchers to perfectly validate the algorithms and techniques.

(vi) For channel estimation, TDD scenarios are only considered for massive MIMO due to the excessive cost of channel estimation and feedback. Even for TDD to work, massive MIMO channel calibration can prove to be a big achievement. New methods and schemes will be needed for the purpose of channel estimation in massive MIMO systems.

(vii) Extremely fast processing algorithms will be required for processing the massive amount of data from the RF chains.

The above-mentioned challenges have been addressed up to some extent in the last few years. To date, many researches on massive MIMO avoided the challenge of considering FDD systems by simply assuming the TDD protocol. The uplink CSI can be more easily obtained at the BS due to the less number of single-antenna users and the strong capability of processing at BS. And then by leveraging the channel reciprocity property, the CSI in the downlink can be directly tacked $[6,7]$. However, due to the fact that radio frequency chains suffer from calibration error and restricted coherence time, the CSI obtained in the uplink may not be correct for the downlink $[8,9]$. Additionally FDD systems have low latency as compared to TDD; therefore the communication is more efficient [10]. Thus, it is of significance to discover the challenging issue of channel estimation for FDD massive MIMO systems, which can assist massive MIMO to be backward attuned with existing FDD dominated cellular networks. There has been extensive analysis on channel estimation for traditional small-scale FDD MIMO systems $[11,12]$. It has been established that the equally spaced and equally powered orthogonal pilots can be ideally suitable to estimate the noncorrelated Rayleigh MIMO channels for single OFDM symbol, where the pilot overhead required increases as the number of transmit antennas increases [11]. The pilot overhead to estimate Rician MIMO channels can be compacted by exploiting the spatial correlation of MIMO channels $[13,14]$. Furthermore, by taking advantage of the temporal channel correlation, more reduced pilot overhead can be attained to estimate MIMO channels linked to multiple OFDM symbols [15-17]. Presently, orthogonal pilots have been extensively introduced in the current MIMO systems; due to small number of transmit antennas (i.e., up to eight antennas in LTE-Advanced system), they have reasonable pilot overhead $[12,18]$. Though, this is a critical issue in massive MIMO systems due to massive figure of antennas at the BS (i.e., up to 128 antennas or even more at the BS [19]). An approach of exploiting the temporal correlation and the delay domain sparsity of channels for achieving the reduced pilot overhead has been presented for FDD massive MIMO systems in [20, 21], but with large number of transmit antennas, the interference cancellation of pilot sequences of different transmit antennas will be difficult. In [22-25] the spatial correlation in sparsity of delay domain MIMO channels is utilized to estimate channels achieving the reduced pilot overhead, but the assumption level of the known channel sparsity to the user is impractical. In [2628], the compressive sensing (CS) based channel estimation schemes are presented by exploiting the spatial channel correlation; however due to having nonideal antenna array, the leveraged spatial correlation can be impaired [2, 8]. In [29] a novel doubly selective channel estimation scheme for MIMOOFDM systems is proposed with both time and frequencydomain training (TFDT) based on structured compressive sensing (SCS). In [30] a new sparse channel model based on dictionary learning is proposed, which adapts to the cell characteristics and promotes a sparse representation. The learned dictionary is able to more robustly and efficiently represent the channel and improve downlink channel estimation accuracy. Furthermore, observing the identical AOA/AOD between the uplink and downlink transmission, a joint uplink and downlink dictionary learning and compressed channel estimation algorithm is proposed to perform downlink channel estimation utilizing information from the simpler uplink training, which further improves downlink channel estimation. In [31] a channel estimator based on block distributed compressive sensing (BDCS) is proposed for the large-scale MIMO systems. BDCS exploits structured sparsity to reduce the pilot overhead. In [32] a new way to estimate sparse channels by construction of beamforming 
dictionary matrices is presented. Continuous basis pursuit (CBP) algorithm that exploits the sparse nature of channels to adaptively estimate the multipath mmWave channels is proposed. In [33] an open-loop and a closed-loop channel estimation scheme for massive MIMO are presented, but the channel statistics cannot be perfectly known to the user in the long term, whereas in conventional broadband wireless communication systems, delay domain channels basically exhibit the sparse nature due to the large channel delay spread and having limited number of major scatterers in the propagation environments $[21,34]$.

In the meantime, MIMO communication systems have similar scatterers in the transmission environment; therefore BS channels associated with one user and several transmit antennas experience similar type of path delays, which shows that these delay domain channels share common sparsity specially in the case of not having very large aperture of antenna array $[2,35]$. Furthermore, throughout the coherence time, such sparsity is nearly unchanged which is due to the fact that path delays fluctuate at very slow rate as compared to path gains because of temporal correlation of channels [36]. In [37] CS based block sparsity adaptive matching pursuit (BSAMP) algorithm is proposed, which targets the estimation of channels of MIMO system with unknown number of channels paths. The BSAMP exploits the joint sparse nature of massive MIMO channels. In [38] CS based probability-weighted subspace pursuit (PWSP) algorithm is proposed, which exploits the probability information attained from previously estimated CIRs to recover the uplink channels in massive MIMO scenario. In [39] such properties of channels in MIMO are considered as the spatiotemporal common sparsity, which is generally ignored in current work. Finally, a general idea of the CS method is presented in [40], in which fundamental setup, recovery techniques, and guarantee of performance are discussed. Furthermore, different subproblems of CS, i.e., sparse approximation, identification of support, and sparse identification, are discussed, with respect to some applications of wireless systems. Design issues of wireless systems, limitations and potentials of CS algorithms, useful tips, and prior information are also discussed to achieve maximum performance. In [40] a valuable guidance is provided for researchers working in the area of wireless communication systems. Before getting into the detail of spatiotemporal common sparsity, we present an overview of compressive sensing.

In this paper we propose a CS based efficient nonorthogonal pilot scheme for massive MIMO communication systems by exploring the temporal and spatial sparsity of massive MIMO channels. And then we propose a channel estimation scheme, i.e., sparsity update CoSaMP (SUCoSaMP), which exploits the temporal and spatial sparsity of massive MIMO channels. The proposed pilot scheme is significantly different from the conventional schemes and substantially reduces the pilot overhead. The proposed pilot scheme employs fully identical subcarriers for pilots of several transmit antennas in a specific antenna group. The antennas placed at base station (BS) are subdivided into groups based on the observation that the coherence time of path gains is inversely proportional to the system carrier frequency whereas the variation in path delays is inversely proportional to the signal bandwidth. Therefore the decision of making antenna groups and determining the number of antennas to be included in one antenna group is taken according to the given system parameters, i.e., systems frequency, system bandwidth, and antenna spacing at BS. Furthermore, considering the antenna array geometry of BS, the proposed nonorthogonal pilot scheme is a space-time adaptive pilot scheme that adaptively changes its design according to the given system parameters. The proposed CS algorithm based channel estimation scheme SUCoSaMP considers the initial sparsity level as 1 and then regularly updates the sparsity level until the stopping criteria are met or a correct sparsity level is achieved in a scenario where sparsity level is unknown. Compared with the conventional CS algorithms SP and OMP and with other available CS based algorithms, the proposed CS based pilot and channel estimation scheme is tested through simulations on systems with different parameters. It was verified that the proposed schemes provided improved channel estimation performance.

The organization of the paper is as follows. Sections 2 and 3 present the details about delay domain spatial and temporal sparsity of massive MIMO communication channels, respectively. Section 4 presents the proposed nonorthogonal pilot scheme based on CS theory. Section 5 illustrates the CS based massive MIMO channel estimation scheme. Section 6 provides the simulation results. And in Section 7 we present the conclusion

Notation. Uppercase and lowercase boldface letters represent the matrices and vectors, respectively. The matrix transpose, inversion, and conjugate transpose are denoted by $(.)^{T},(.)^{-1}$, and $(.)^{*}$, respectively. The Moore-Penrose inversion of matrix is denoted by $(.)^{\dagger}$. The $l_{2}$-norm operation and Frobeniusnorm operation are denoted by $\|.\|_{2}$ and $\|.\|_{F}$, respectively. The cardinality of a set and the $\mathrm{j}$-th column vector of the matrix $\psi$ are denoted by $|\cdot|_{C}$ and $\psi^{(j)}$, respectively. Finally the complementary set of the set $T$ and the integer floor operator are denoted by $T^{C}$ and $\lfloor$.$\rfloor , respectively.$

\section{Delay Domain Spatial Sparsity}

Considerable experimental researches have discovered that, in delay domain massive MIMO, channels demonstrate spatial sparsity. This is due to the fact that the number of significant scatterers is limited in wireless communication in fading environments, while in communication between base station (BS) and users, the transmission distance is very large as compared to the distance between several antennas in an antenna array placed at BS. That is to say, CIRs associated with several transmit antennas and one user exhibit similar path delays; therefore they also share identical common support of CIRs.

Consider a massive MIMO-OFDM system where $M$ transmit antennas are placed at BS. The CIR between $\mathrm{m}$-th transmit antenna and one single-antenna user for z-th OFDM symbol is expressed by

$$
\boldsymbol{d}_{z, m}=\left[d_{z, m}(1), d_{z, m}(2), \ldots, d_{m, r}(L)\right]^{T}
$$




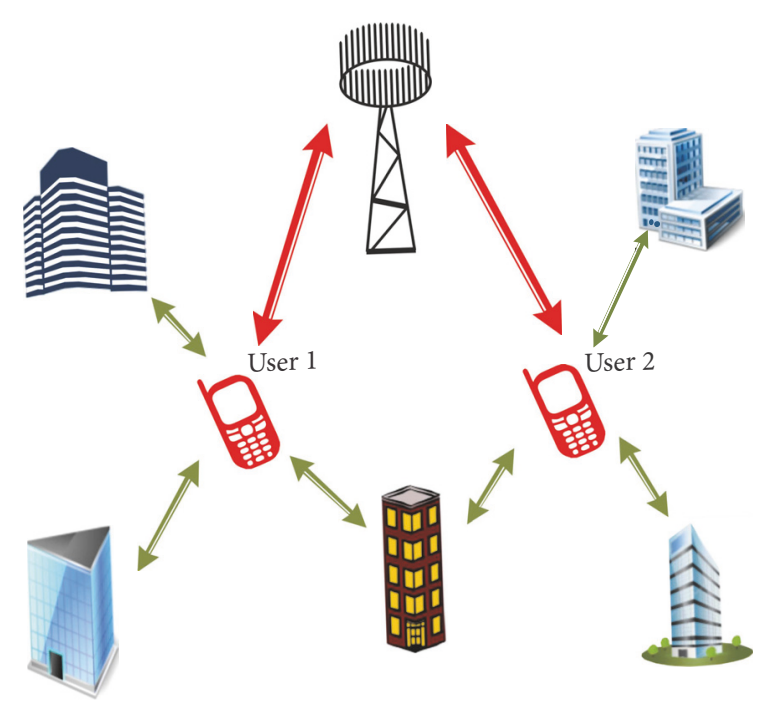

(a)

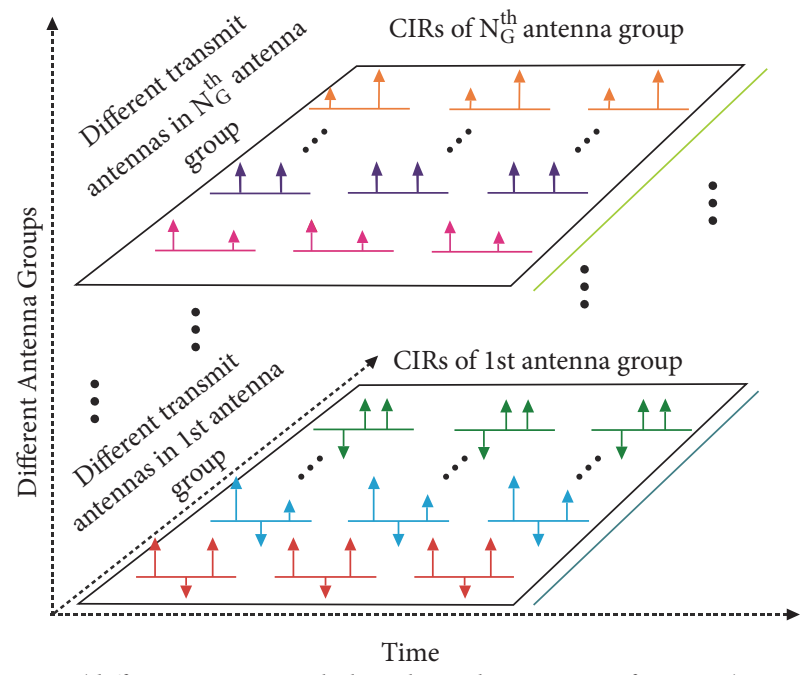

(different OFDM symbols within coherence time for user 1)

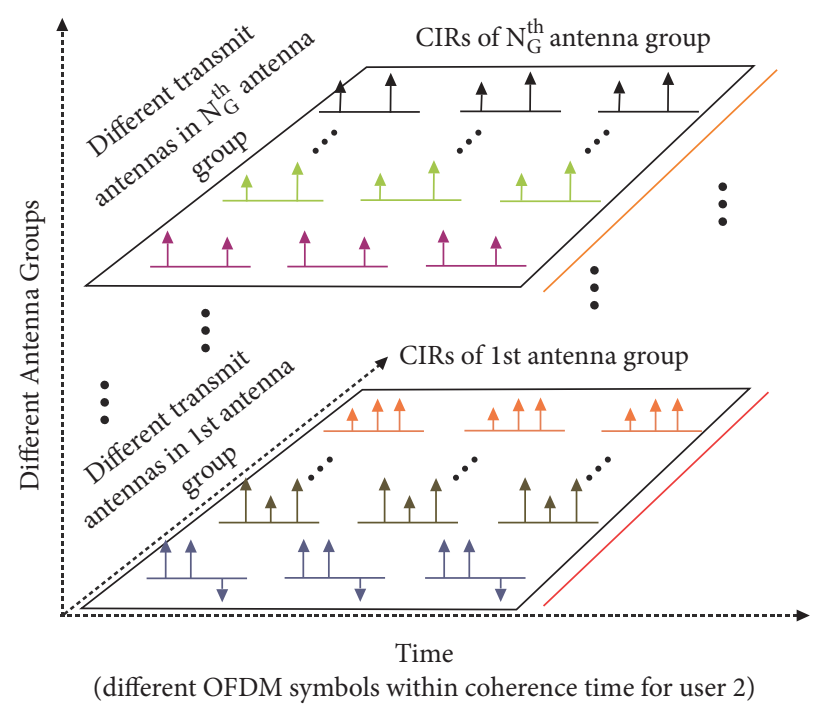

(b)

FIGURE 1: Delay domain spatial and temporal sparsity of massive MIMO channels: (a) limited number of scatterers and common scatterers in wireless communication scenario; (b) spatial and temporal sparsity of massive MIMO channels in delay domain between two users and colocated antenna array.

where $1 \leq m \leq M$ and $L$ is the channel length equivalent to the maximum delay spread. Let $S_{z, m}$ be the sparsity level of CIR between one transmit-receive antenna pair, i.e., the number of nonzero elements in $\boldsymbol{d}_{z, m}$; the support of $\boldsymbol{d}_{z, m}$ can be expressed as

$$
\begin{aligned}
& P_{z, m}=\operatorname{supp}\left\{\boldsymbol{d}_{z, m}\right\}=\left\{l:\left|d_{z, m}[l]\right|>0\right\} \\
& \text { with } 1 \leq l \leq L
\end{aligned}
$$

where $S_{z, m}=\left|P_{z, m}\right|_{C}$ fulfilling $S_{z, m} \ll L$. And due to spatial sparsity we have the following.

$$
P_{z, 1}=P_{z, 2}=\cdots=P_{z, M}
$$

The delay domain spatial sparsity with specific system parameters will be detailed in Section 5 .
Figure 1 shows the common sparse pattern of CIRs for different transmit-receive antenna pairs.

\section{Delay Domain Temporal Sparsity}

In [39], the authors have discovered that fast time-varying channels illustrate temporal correlation. That is to say, the variations in path gains are significant whereas the path delays are almost invariant for various consecutive OFDM symbols. The reason is that path delays variation duration over time-varying channels is inversely proportional to the signal bandwidth while the coherence time of path gains is inversely proportional to carrier frequency of the system [28]. 
Consider a system with signal bandwidth $B=10 \mathrm{MHz}$ and carrier frequency of system $f_{c}=2 \mathrm{GHz}$; the path delays vary much slower than the path gains [11]. Therefore, due to the nearly invariant path delays, the CIRs share the common sparsity for $R$ adjacent OFDM symbols over the coherence time of path delays. The supports of CIRs associated with $R$ successive OFDM symbols signify the following.

$$
P_{1, m}=P_{z, m}=\cdots=P_{R, m}, \quad 1 \leq m \leq M
$$

Figure 1 demonstrates the temporal sparsity of massive MIMO channels.

\section{Proposed Nonorthogonal Pilot Scheme}

In this section we shall propose a nonorthogonal pilot scheme based on the above-mentioned two observations. First the basic idea to divide the antennas placed at BS into subgroups is proposed. The proposed scheme of dividing the antennas into subgroups is based on system parameters and the temporal and spatial sparsity of massive MIMO channels. Then after creating the antenna groups, a specific nonorthogonal pilot scheme is proposed.

4.1. Proposed Scheme for Creating the Antenna Groups. There will be the uniform distribution of antennas to be included in subgroups. Consider a system with signal bandwidth $\mathrm{B}$, system carrier frequency $f_{c}$, total number of antennas placed at the BS in a uniform linear antenna array $M$, number of subgroups $N_{g}$, and number of antennas in one group $M_{n g}$. The maximum resolvable distance $D_{\max }$ must be smaller than c/10B [38]; that is to say, to have two channel taps resolvable, the time interval of arrival must be less than $1 / 10 \mathrm{~B}$, where $\mathrm{c}$ is the speed of light [38]. The two successive antennas are spaced by the distance $d_{m}=\lambda / 2$; therefore the maximum distance between two antennas can be $d_{T}=d_{m}(M-1)$ and the maximum distance between two antennas in a single group can be $d_{M_{n g}}=d_{m}\left(M_{n g}-1\right)$. In order to guarantee the spatial sparsity and based on the condition that $D_{\max } / c<1 / 10 B$, the $d_{M_{n g}}$ must satisfy $d_{M_{n g}}<D_{\max }$.

And the formula for number of antennas $M_{n g}$ in each subgroup $N_{g}$ can be derived as follows:

$$
\begin{aligned}
d_{m}\left(M_{n g}-1\right) & \leq \frac{c}{10 B} \\
\frac{\lambda}{2\left(M_{n g}-1\right)} & \leq \frac{c}{10 B} \\
M_{n g} & \leq \frac{c}{5 B\left(c / f_{c}\right)}+1
\end{aligned}
$$

which clearly shows that $M_{n g}$ is a function of $B$ and $f_{c}$. Therefore the $N_{g}$ can be given by the following.

$$
N_{g}=\left\lfloor\frac{M}{M_{n g}}\right\rfloor+\alpha
$$

The constant $\alpha$ represents an integer to make sure the uniform distribution of antennas in each subgroup, i.e.,

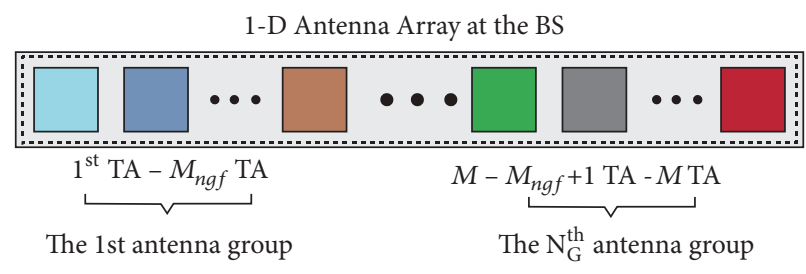

FIGURE 2: 1D antenna array at the BS.

$M$, is completely divisible by $N_{g}$. Similarly actual number of antennas $M_{n g f}$ in each group can be calculated by the following.

$$
M_{n g f}=\frac{M}{N_{g}}
$$

The formula for $N_{g}$ will ensure the spatial sparsity for any massive MIMO system with large $1 \mathrm{D}$ antenna array placed at BS. Moreover, since the $M_{n g}$ is a function of system parameters $\mathrm{B}$ and $f_{c}$, if the $\mathrm{B}$ and $f_{c}$ are changed, the $N_{g}$ still ensures the spatial sparsity for antennas. And also $N_{g}$ is the minimum number of subgroups a system must have to ensure spatial sparsity; moreover by increasing $N_{g}$, spatial sparsity of system will remain preserved. For example, consider a system with the following specifications:

$M=1281 \mathrm{D}$ antenna array, $f_{c}=2 \mathrm{GHz}, \mathrm{B}=20 \mathrm{MHz}$, $M_{n g}$ given in (7) can be calculated as $21, N_{g}$ will be equal to 8 with $\alpha=2$, and $M_{n g f}$ will be equal to 16 . Based on these calculations the initial condition is satisfied, that is, $d_{M_{n g}}<D_{\text {max }}$; hence the spatial sparsity is preserved for the system.

The $N_{g}$ antenna groups are shown in Figure 2, where TA denotes the transmit antenna.

In Section 4.2, first we will derive a massive MIMO system model. And then specific pilot design will be proposed in Section 4.3.

4.2. Massive MIMO System Model. Consider a massive MIMO-OFDM system with $M$ transmit antennas placed at BS communicating with one user; $\xi_{n}$ represents the index set of pilot subcarriers for n-th antenna group; the choice of $\xi_{n}$ will be detailed in Section 4.3). There are total $N$ subcarriers in one OFDM symbol, out of which $N_{p}$ corresponds to pilot subcarriers and the pilot sequence of $\mathrm{m}$-th transmit antenna in $\mathrm{n}$-th antenna group is denoted by $\mathbf{s}_{\mathrm{m}, \mathrm{n}} \in \mathbb{C}^{N_{p} \mathrm{x}} \cdot \mathbf{y}_{\mathrm{z}, \mathrm{n}} \in$ $\mathbb{C}^{N_{p} \mathrm{xl}}$ is the received vector of the pilot sequence of $\mathrm{z}$-th OFDM symbol of n-th antenna group at user for $N_{g}$ antenna groups; $\mathbf{y}_{\mathrm{z}, \mathrm{n}}$ can be expressed as

$$
\mathbf{y}_{\mathrm{z}, \mathrm{n}}=\left.\sum_{m=1}^{M_{\text {ngf }}} \operatorname{diag}\left\{\mathbf{s}_{\mathrm{m}, \mathrm{n}}\right\} \mathbf{R}\right|_{\xi_{n}, \mathrm{n}}\left[\begin{array}{c}
\mathbf{d}_{\mathrm{m}, \mathrm{z}, \mathrm{n}} \\
\mathbf{0}_{(N-L) \times 1}
\end{array}\right]+\mathbf{w}_{\mathrm{z}, \mathrm{n}}
$$

with $1<\mathrm{n}<N_{g}$

$$
\mathbf{y}_{\mathrm{z}, \mathrm{n}}=\left.\sum_{m=1}^{M_{n g f}} \mathbf{S}_{\mathrm{m}, \mathrm{n}} \mathbf{R}_{\mathbf{L}}\right|_{\xi_{n}, \mathrm{n}} \mathbf{d}_{\mathrm{m}, \mathrm{z}, \mathrm{n}}+\mathbf{w}_{\mathrm{z}, \mathrm{n}}
$$




$$
=\sum_{m=1}^{M_{n g f}} \psi_{\mathrm{m}, \mathrm{n}} \mathbf{d}_{\mathrm{m}, \mathrm{z}, \mathrm{n}}+\mathbf{w}_{\mathrm{z}, \mathrm{n}}
$$

where $\mathbf{R} \in \mathbb{C}^{N \mathrm{xN}}$ is the Discrete Fourier Transform matrix (DFT), $\mathbf{y}_{z, n}$ is expressed after exclusion of guard interval, $\mathbf{d}_{\mathrm{m}, \mathrm{z}, \mathrm{n}} \in \mathbb{C}^{L \mathrm{x} 1}$ is the CIR vector of $\mathrm{m}_{\mathrm{th}}$ transmit antenna of $\mathrm{n}$-th antenna group for $\mathrm{z}_{\mathrm{th}}$ OFDM symbol, $\left.\mathbf{R}\right|_{\xi_{n}, \mathrm{n}} \in \mathbb{C}^{N_{p} \mathrm{xN}}$ is a submatrix comprised of $N_{p}$ rows selected according to $\xi_{n},\left.\mathbf{R}_{\mathbf{L}}\right|_{\xi_{n}, \mathrm{n}} \in \mathbb{C}^{N_{p} \times L}$ contains the first L columns of $\left.\mathbf{R}\right|_{\xi_{n}, \mathrm{n}} \in$ $\mathbb{C}^{N_{p} \mathrm{x} N}, \mathbf{S}_{\mathrm{m}, \mathrm{n}}=\operatorname{diag}\left\{\mathbf{s}_{\mathrm{m}, \mathrm{n}}\right\} \in \mathbb{C}^{N_{p} \mathrm{x} N_{p}}, \psi_{\mathrm{m}, \mathrm{n}}=\left.\mathbf{S}_{\mathrm{m}, \mathrm{n}} \mathbf{R}_{\mathbf{L}}\right|_{\xi_{n}, \mathrm{n}} \in$ $\mathbb{C}^{N_{p} \mathrm{x} L}$, and $\mathbf{w}_{\mathrm{z}, \mathrm{n}}$ represents the Additive White Gaussian Noise of $\mathrm{n}$-th antenna group for $\mathrm{z}$-th OFDM symbol.

The equation can be rearranged as

$$
\mathbf{y}_{\mathrm{z}, \mathrm{n}}=\psi_{\mathrm{n}} \widetilde{\mathbf{d}}_{\mathrm{z}, \mathrm{n}}+\mathbf{w}_{\mathrm{z}, \mathrm{n}}
$$

where $\psi_{\mathrm{n}}=\left[\psi_{1, \mathrm{n}}, \psi_{2, \mathrm{n}}, \ldots, \psi_{M_{n g f}, \mathrm{n}}\right] \in \mathbb{C}^{N p \times M_{n g f} L}$ and the aggregate CIR vector of $\mathrm{n}$-th antenna group is given by $\widetilde{\mathbf{d}}_{\mathrm{z}, \mathrm{n}}=$ $\left[\mathbf{d}_{1, \mathrm{z}, \mathrm{n}}, \mathbf{d}_{2, \mathrm{z}, \mathrm{n}}, \ldots, \mathbf{d}_{M_{n g f}, \mathrm{z}, \mathrm{n}}\right]^{\mathrm{T}} \in \mathbb{C}^{M_{n g f} L \times 1}$. As explained earlier, the CIR vector $\mathbf{d}_{\mathrm{m}, \mathrm{z}, \mathrm{n}}$ exhibits spatial and temporal sparsity; therefore $\widetilde{\mathbf{d}}_{\mathrm{z}, \mathrm{n}}$ is also a sparse signal. The system in equation is an underdetermined system due to the fact that $N_{p}<M_{n g f} L$ and cannot be reliably solved using the traditional channel estimation methods [39].

Moreover the equation can be rearranged into structured sparse form according to [39] as

$$
\mathbf{y}_{\mathrm{z}, \mathrm{n}}=\mathbf{A}_{\mathrm{n}} \tilde{\mathbf{h}}_{\mathrm{z}, \mathrm{n}}+\mathbf{w}_{\mathrm{z}, \mathrm{n}}
$$

where $\widetilde{\mathbf{h}}_{\mathrm{z}, \mathrm{n}}=\left[\mathbf{h}_{1, \mathrm{z}, \mathrm{n}}^{\mathrm{T}}, \mathbf{h}_{2, \mathrm{z}, \mathrm{n}}^{\mathrm{T}}, \ldots, \mathbf{d}_{\mathrm{L}, \mathrm{z}, \mathrm{n}}^{\mathrm{T}}\right]^{\mathrm{T}} \in \mathbb{C}^{M_{n g f} L \times 1}$ is a structured sparse equivalent CIR vector; $\mathbf{h}_{\mathrm{l,z, \textrm {n }}}=\left[d_{1, \mathrm{z}, \mathrm{n}}[l], d_{2, \mathrm{z}, \mathrm{n}}[l]\right.$, $\left.\ldots, d_{M_{n g}, \mathrm{z}, \mathrm{n}}[l]\right]^{\mathrm{T}}$ for $1 \leq l \leq L$ and after reformulation $\psi_{\mathrm{n}}$ can be converted into $\mathbf{A}_{\mathrm{n}}$, where $\mathbf{A}_{\mathrm{n}}$ can be written as

$$
\mathbf{A}_{\mathrm{n}}=\left[\mathbf{A}_{1, \mathrm{n}}, \mathbf{A}_{2, \mathrm{n}}, \ldots \mathbf{A}_{\mathrm{L}, \mathrm{n}}\right] \in \mathbb{C}^{N_{p} \times M_{n g f} L}
$$

where $\mathbf{A}_{l, n}=\left[\psi_{1, n}^{(l)}, \psi_{2, n}^{(l)}, \ldots, \psi_{M_{n g f}, n}^{(l)}\right]=\left[A_{1, n, l}, A_{2, n, l}, \ldots\right.$ $\left.\mathrm{A}_{M_{n g f}, n, l}\right] \in \mathbb{C}^{N p \times M_{n g f}}$.

After converting to structured form, we will have $N_{g}$ equations to be solved simultaneously corresponding to each subgroup. The received pilot vectors of $\mathrm{z}_{\mathrm{th}}$ OFDM symbol for $N_{g}$ subgroups can be expressed as follows.

$$
\begin{gathered}
\mathbf{y}_{\mathrm{z}, 1}=\mathbf{A}_{1} \widetilde{\mathbf{h}}_{\mathrm{z}, 1}+\mathbf{w}_{\mathrm{z}, 1} \\
\mathbf{y}_{\mathrm{z}, 2}=\mathbf{A}_{2} \widetilde{\mathbf{h}}_{\mathrm{z}, 2}+\mathbf{w}_{\mathrm{z}, 2} \\
\vdots \\
\mathbf{y}_{\mathrm{z}, \mathrm{n}}=\mathbf{A}_{\mathrm{n}} \widetilde{\mathbf{h}}_{\mathrm{z}, \mathrm{n}}+\mathbf{w}_{\mathrm{z}, \mathrm{n}} \\
\vdots \\
\mathbf{y}_{\mathrm{z}, N_{g}}=\mathbf{A}_{N_{g}} \widetilde{\mathbf{h}}_{\mathrm{z}, N_{g}}+\mathbf{w}_{\mathrm{z}, N_{g}}
\end{gathered}
$$

The system equation of each subgroup exhibits structured sparsity, which provides the motivation to apply CS theory to recover the high dimension structured sparse signal $\widetilde{\mathbf{h}}_{\mathrm{z}, N_{g}}$ from the low dimension received pilot vector $\mathbf{y}_{\mathrm{z}, N_{g}}$. The CS based sparse signal recovery/channel estimation will be explained in Section 5.

4.3. Proposed Pilot Design. Mostly wireless communication systems detect the data with the help of pilot signals. Specifically the purpose of pilot signals is to assist the receiver in estimating the wireless channels and then the receiver coherently detects the data on the basis of estimated channel [41].

In massive MIMO communication systems, due to large number of transmit antennas at BS, the number of channels gets prohibitively high and thus results in high pilot overhead for estimation of these channels. Therefore there is a need for methods to reduce the high pilot overhead in massive MIMO communication systems to achieve the target of high data rate.

In this research paper CS theory is applied to reduce the high pilot overhead based on the fact that the wireless channels undergo spatial and temporal sparsity. CS based pilot design significantly reduces the pilot overhead as compare to conventional pilot design. Figure 3 demonstrated the proposed, uniformly distributed, and identical pilot subcarrier for multiple antennas, while Figure 4 shows the convention pilot design (i.e., orthogonal pilots for different antennas). The proposed pilot design allows the system to provide more resources to data.

The CS based pilot design permits the antennas of each subgroup to occupy identical subcarriers for pilots within a group, while pilot subcarriers for each subgroup are completely different from each other as shown in Figure 3. The design is based on the choice of $\xi_{n}$. The guard interval can be calculated by $N_{G}=N / B x($ maximum channel delay spread $)$. Consider a set $O=\left\{1,2, \ldots, N-N_{G}\right\}$. The $\xi_{n}$ is a subset of $O$, represents the index of pilot subcarriers for n-th subgroup, and is identical for all the antennas within that group; the formula for creating the $\xi_{n}$ can be given by

$$
\begin{aligned}
\xi_{n} & =\left\{n+\text { spacing } * q:\left\lfloor\frac{M}{M_{n g}}\right\rfloor-\alpha \leq N_{g}\right. \\
& \leq \text { spacing, } 0<q<N_{p}-1 \text { and sapcing } \\
& \left.=\left\lfloor\frac{N-N_{G}}{N_{p}}\right\rfloor \geq\left\lfloor\frac{M}{M_{n g}}\right\rfloor-\alpha\right\}
\end{aligned}
$$

where $N_{p}>L$ and by solving the inequality $\left\lfloor\left(N-N_{G}\right) / N_{p}\right\rfloor \geq$ $\left\lfloor M / M_{n g}\right\rfloor-\alpha$ we have the following.

$$
M_{n g f} \leq N_{p} \leq \frac{\left(N-N_{G}\right)}{\left(M-\alpha M_{n g}\right)} M_{n g}
$$

There are $N_{G}$ unique sets associated with $\xi_{n}$, i.e., $\xi_{1} \ldots .$. $\xi_{n} \ldots \xi_{N g}$. There will be total $N_{g} N_{p}$ subcarriers occupied 

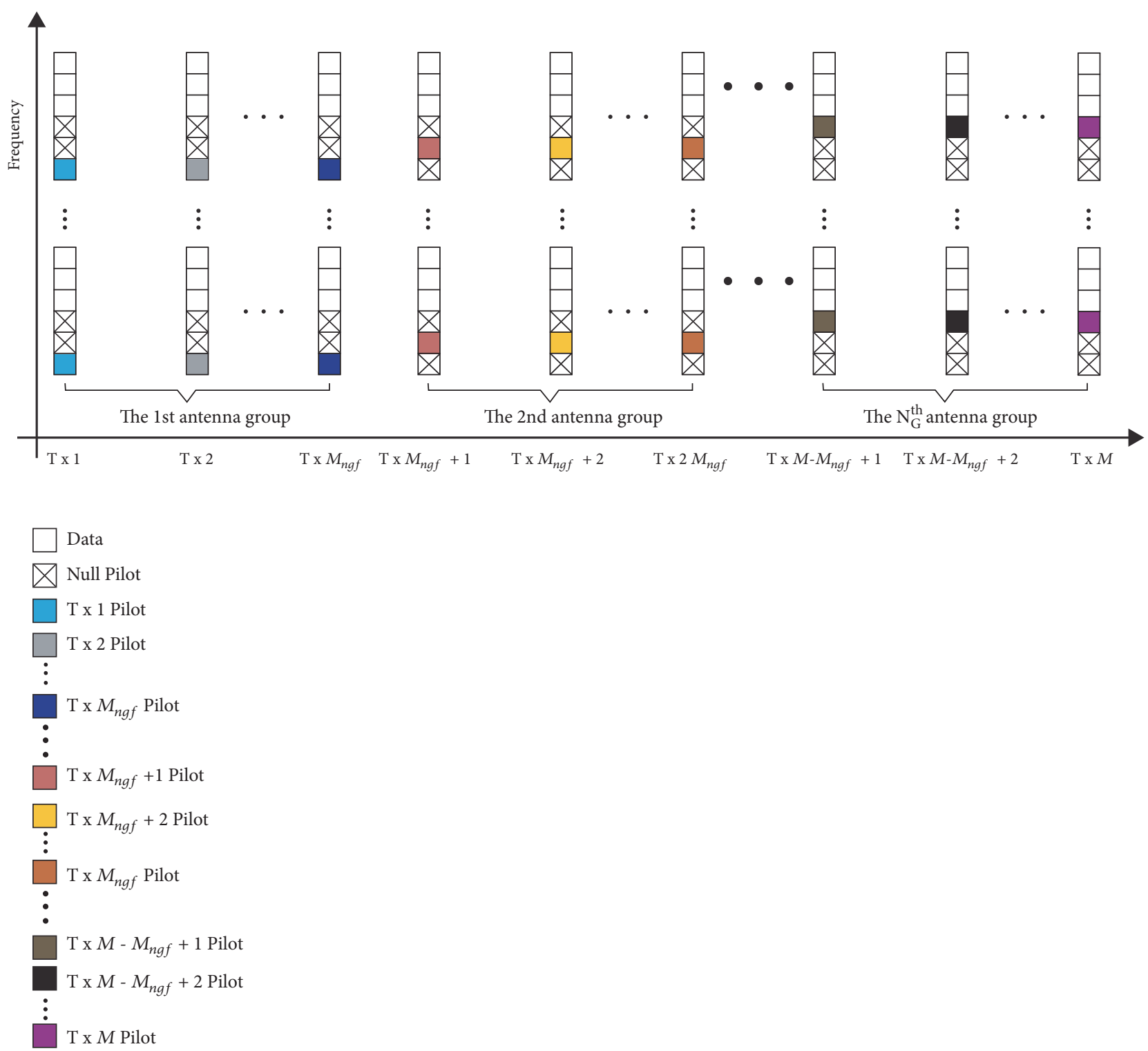

Figure 3: Proposed pilot design.

for pilot vectors for $M$ transmit antennas at BS. Figure 3 demonstrates the proposed pilot design.

$N_{p}=\left|\xi_{n}\right|_{C}$ is the number of subcarriers for pilot vector in OFDM symbol of $\mathrm{n}_{\text {th }}$ subgroup.

Pilot subcarriers of $\mathrm{n}_{\text {th }}$ group are the null pilots for all the other $N_{g}-1$ subgroups whereas $N-N_{G}-N_{g} N_{p}$ subcarriers are available for data.

Pilot overhead ratio is defined by $\beta_{p}=N_{g} N_{p} / N$.

Figure 4 shows the conventional pilots in which different pilots are allocated to different antennas resulting in very high pilot overhead.

\section{Massive MIMO Channel Estimation Based on Compressive Sensing}

The basic idea presented by CS theory is to recover a signal which is sparse in some domain from extremely small amount of nonadaptive linear measurements by applying convex optimization. In a different opinion, it relates the precise recovery of a sparse vector of high dimension by reducing its dimension. From another point of view, the problem can be considered as calculation of a signal's sparse coefficient with respect to an overcomplete system. The concept of compressed sensing was primarily applied for random sensing matrices, which allow for a reduced amount of nonadaptive, linear measurements. These days, the idea of compressed sensing has been generally replaced by sparse recovery.

In (13) it can be seen that $\widetilde{\mathbf{h}}_{\mathrm{z}, \mathrm{n}}$ demonstrates structured sparsity in delay domain, where $\widetilde{\mathbf{h}}_{z, \mathrm{n}}$ is $S_{z, m, n}$-sparse vector due to

$$
P_{z, m, n}=\operatorname{supp}\left\{\tilde{\mathbf{h}}_{z, n}\right\}=\left\{l:\left|\tilde{h}_{z, n}[l]\right|>0\right\}
$$



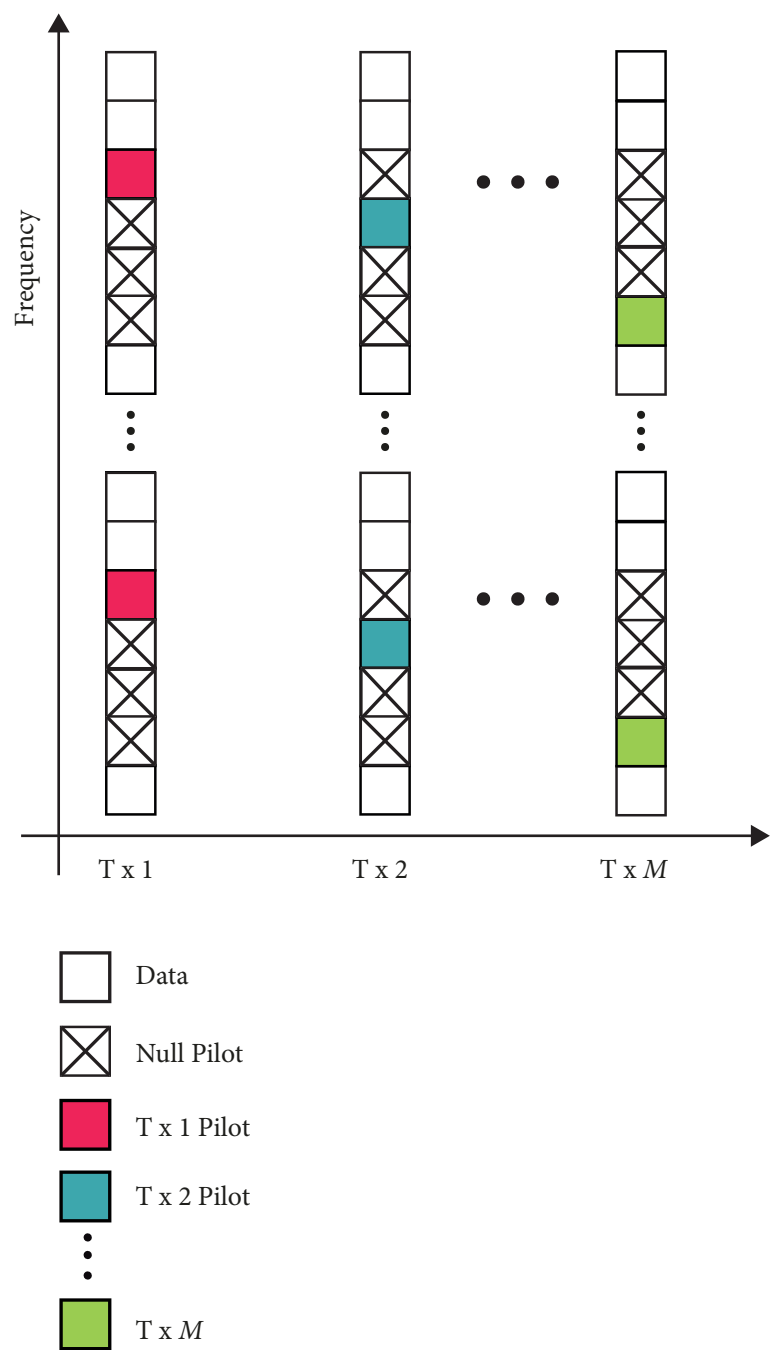

FIgURE 4: Conventional orthogonal pilot design.

where $S_{z, m, n}=\left|P_{z, m, n}\right|_{C}$ fulfilling $S_{z, m, n} \ll L$. And due to spatial sparsity we have the following.

$$
P_{z, 1, n}=P_{z, 2, n}=\cdots=P_{z, M, n}
$$

The desired small correlation of $\mathbf{A}_{\mathrm{n}}$ according to CS theory is achieved which is described in [39]; therefore reliable sparse recovery is guaranteed. It is further shown in [39] that any two columns of $\mathbf{A}_{\mathrm{n}}$ attain excellent cross correlation between them according to RMT since pilot design proposed in [39] is the special case of proposed pilot design. Therefore the proposed pilot design is simple to employ and also supportive in terms of compatibility with current wireless networks [39].

For $K$ adjacent OFDM symbols having identical pattern of pilots, we have

$$
\mathbf{Y}_{\mathrm{n}}=\mathbf{A}_{\mathrm{n}} \mathbf{H}_{\mathrm{n}}+\mathbf{W}_{\mathrm{n}}
$$

where $\mathbf{Y}_{\mathrm{n}}=\left[\mathbf{y}_{\mathrm{z}, \mathrm{n}}, \mathbf{y}_{\mathrm{z}+1, \mathrm{n}}, \ldots, \mathbf{y}_{\mathrm{z}+K-1, \mathrm{n}}\right] \mathbb{C}^{N p \times K}$.
The $\mathbf{A}_{\mathrm{n}}$ derived in massive MIMO model satisfies the Structure Restricted Isometric Property (SRIP) condition. Specifically SRIP can be given by the following.

$$
\sqrt{1-\delta}\left\|\mathbf{A}_{N_{g}}\right\|_{F} \leq\left\|\mathbf{A}_{\mathrm{n}} \tilde{\mathbf{h}}_{\mathrm{z}, \mathrm{n}}\right\|_{F} \sqrt{1-\delta}\left\|\mathbf{A}_{N_{g}}\right\|_{F}
$$

The definition and justification for SRIP are discussed in detail in [39], where $\delta \in[0,1)$.

Our aim is to recover $\widetilde{\mathbf{h}}_{\mathrm{z}, \mathrm{n}}$, given $\mathbf{y}_{\mathrm{z}, \mathrm{n}}$. Under the framework of CS theory, the massive MIMO channels $\tilde{\mathbf{h}}_{\mathrm{z}, \mathrm{n}}$ are estimated by means of the following problem:

$$
\begin{aligned}
\widehat{\mathbf{h}}=\arg \min & \left\|\widetilde{\mathbf{h}}_{\mathrm{z}, \mathrm{n}}\right\|_{1} \\
\text { s.t. } & \left\|\mathbf{y}_{\mathrm{z}, \mathrm{n}}-\mathbf{A}_{\mathrm{n}} \widetilde{\mathbf{h}}_{\mathrm{z}, \mathrm{n}}\right\|_{2}<\epsilon
\end{aligned}
$$

where $\epsilon$ is the noise variance. There are many algorithms that can solve the problem. For example, projected gradient methods or interior point methods can be used for applying convex optimization. A famous greedy algorithm is orthogonal matching pursuit (OMP). 
Input: Sensing matrix $\mathbf{A}_{n}$ and noisy measurement vector $\mathbf{y}_{\mathrm{z}, \mathrm{n}}$

Output: An sparse estimation $\widehat{\mathbf{h}}$ of channels $\left\{\mathbf{d}_{\mathrm{m}, \mathrm{z}, \mathrm{n}}\right\}_{m=1, n=1}^{m=M_{n g}, n=N_{g}}$

Step 1 (Initialization)

1. $\check{\mathbf{h}}^{0} \longleftarrow 0$

Trivial initial approximation

2. $\quad v \longleftarrow \mathrm{y}_{\mathrm{z}, \mathrm{n}}$

Current samples $=$ input samples

3. $k \longleftarrow 0$

Iterative index

4. $s \longleftarrow 1$

Initial sparsity level

Step 2 Solve the structure sparse vector $\widetilde{\mathbf{h}}_{\mathrm{z}, \mathrm{n}}$ to (15)

Repeat

1. $k \longleftarrow k+1$

2. $y \longleftarrow \mathbf{A}_{\mathrm{n}}^{*} \mathbf{v}$

Make the signal proxy

3. $\Omega \longleftarrow \operatorname{supp}\left(\mathbf{y}_{2 \mathrm{~s}}\right)$

Identify large components

4. $\mathrm{T} \longleftarrow \Omega \cup \operatorname{supp}\left(\check{\mathbf{h}}^{k-1}\right)$

Merge supports

5. $\left.\breve{\boldsymbol{h}}\right|_{T} \longleftarrow A_{n T}^{\dagger} \mathbf{y}_{\mathrm{z}, \mathrm{n}}$

Signal estimation by least squares

6. $\left.\breve{\boldsymbol{h}}\right|_{T^{C}} \longleftarrow 0$

7. $\check{\mathbf{h}}^{k} \longleftarrow \breve{\boldsymbol{h}}_{s}$

Prune to get next approximation

8. $\quad \boldsymbol{v} \longleftarrow \mathbf{y}_{z, \mathrm{n}}-\mathbf{A}_{n} \check{\mathbf{h}}^{k}$

Update the current samples

if $\left\|\boldsymbol{v}^{k}\right\|_{2}<\left\|\boldsymbol{v}^{k+1}\right\|_{2}$

9. Iteration with fixed sparsity level

else

10. Update sparsity level $\check{\mathbf{h}}_{s} \longleftarrow \check{\mathbf{h}}^{k} ; \boldsymbol{v}_{s} \longleftarrow \boldsymbol{v}^{k} ; s \longleftarrow s+1$

end if

Until stopping criterion true

Step 3 Obtain channels $\widehat{\boldsymbol{h}}=\check{\mathbf{h}}_{s-1}$ and obtain estimation of channels $\left\{\mathbf{d}_{\mathrm{m}, \mathrm{z}, \mathrm{n}}\right\}_{m=1, n=1}^{\boldsymbol{m}=M_{n g f}, n=N_{g}}$ according to (11)-(15)

Algorithm 1: Proposed SUCoSaMPrecovery algorithm.

For channel estimation purpose we propose the SUCoSaMP algorithm derived from basic CoSaMP as described in Algorithm 1. There will be $N_{g}$ similar parallel processing required for estimating the massive MIMO channels with $N_{g}$ sub-antenna groups; i.e., the same algorithm will be working simultaneously with user to estimate channels of $N_{g}$ subgroups.

There are many natural approaches of stopping the algorithm. We follow the following stopping criterion: if $\left\|\boldsymbol{v}^{k+1}\right\|_{2}>$ $\left\|\boldsymbol{v}_{s-1}\right\|_{2}$, the iteration is stopped [39]. The information of correct sparsity level $S_{z, m, n}$ is usually not available and also it is practically not possible to have prior knowledge of correct sparsity level, whereas information about sparsity level plays a significant role in compressive sensing problem of solving underdetermined system and it is also required as prior information by most of the CS based algorithms. The proposed SUCoSaMP algorithm does not require prior information of sparsity level because it adaptively acquires the sparsity level and avoids the unrealistic assumption of having prior information of correct sparsity level.
In steps 2.1 to 2.9 the target of SUCoSaMP algorithm is to obtain the solution $\widetilde{\mathbf{h}}_{\mathrm{z}, \mathrm{n}}$ to (15) with fixed sparsity level $s$ similar to conventional CoSaMP. The condition $\left\|\boldsymbol{v}^{k}\right\|_{2} \leq$ $\left\|\boldsymbol{v}^{k+1}\right\|_{2}$ shows that the solution $\widetilde{\mathbf{h}}_{\mathrm{z}, \mathrm{n}}$ to (15) has been acquired and then the new iteration is started with updated sparsity level $s+1$. This process is repeated until the stopping criteria are true and then the iteration is stopped. We get the solution to (15) with updated sparsity level and obtain the channels, i.e., $\widehat{\boldsymbol{h}}=\check{\mathbf{h}}_{s-1}$.

Note that the proposed SUCoSaMP algorithm works in the same way as the CoSaMP algorithm. The CoSaMP algorithm is basically based on basic OMP. The SUCoSaMP algorithm has incorporated some other ideas from the literature to present strong guarantees that OMP and CoSaMP cannot provide and to speed up the algorithm as compared to OMP.

The major advantage of SUCoSaMP over OMP, CoSaMP, and basic subspace pursuit (SP) algorithms is that it does not require prior knowledge of sparsity level which is an 
TABLE 1: Major steps involved in CoSaMP and SUCoSaMP.

\begin{tabular}{|c|c|c|}
\hline & CoSaMP & SUCoSaMP \\
\hline 1. Classification. & $\begin{array}{l}\text { The algorithm creates a replacement of the residual from the existing samples and } \\
\text { places the biggest components of the replacement. }\end{array}$ & $\begin{array}{l}\text { Follows the same step of } \\
\text { CoSaMP. }\end{array}$ \\
\hline 2. Support union. & $\begin{array}{l}\text { The set of recently recognized components is joined with the set of components that } \\
\text { emerge in the present approximation. }\end{array}$ & $\begin{array}{l}\text { Follows the same step of } \\
\text { CoSaMP. }\end{array}$ \\
\hline 3. Estimation. & $\begin{array}{l}\text { The algorithm finds the solution of a least-squares problem to estimate the objective } \\
\text { signal on the combined set of components. }\end{array}$ & $\begin{array}{l}\text { Follows the same step of } \\
\text { CoSaMP. }\end{array}$ \\
\hline 4. Pruning. & $\begin{array}{l}\text { The algorithm generates a fresh estimation by keeping only the biggest entries in } \\
\text { this least-squares approximation of signal. }\end{array}$ & $\begin{array}{l}\text { Follows the same step of } \\
\text { CoSaMP. }\end{array}$ \\
\hline 5. Sample Update. & $\begin{array}{l}\text { Finally, the algorithm updates the samples such that they reflect the residual, the } \\
\text { un-approximated elements of the signal. }\end{array}$ & $\begin{array}{l}\text { Follows the same step of } \\
\text { CoSaMP. }\end{array}$ \\
\hline 6. Stopping Criterion & Until stopping criterion is true. & $\begin{array}{l}\text { Until first stopping } \\
\text { criterion is true. }\end{array}$ \\
\hline $\begin{array}{l}\text { 7. Sparsity level } \\
\text { Update }\end{array}$ & None & $\begin{array}{l}\text { The algorithm updates the } \\
\text { sparsity level }\end{array}$ \\
\hline 8. Stopping Criterion & None & $\begin{array}{l}\text { Until second stopping } \\
\text { criterion is true. }\end{array}$ \\
\hline
\end{tabular}

TABLE 2: CoSaMP and SUCoSaMP hypothesis.

\begin{tabular}{|c|c|c|}
\hline & CoSaMP & SUCoSaMP \\
\hline 1. & $\begin{array}{l}\text { The sparsity level s is fixed. (i.e. initialization with fixed value of } \\
\text { sparsity level). }\end{array}$ & $\begin{array}{l}\text { The sparsity level s is not fixed (i.e. initialization with sparsity } \\
\text { level equals 1). It adaptively acquires the correct sparsity level. }\end{array}$ \\
\hline 2. & $\begin{array}{l}\text { The sensing matrix } \mathbf{A}_{n} \text { has restricted isometry constant } \\
\qquad \delta_{4 s} \leq 0.1\end{array}$ & $\begin{array}{l}\mathbf{A}_{n} \text { satisfies the Structure Restricted Isometric Property (SRIP) } \\
\text { condition according to [39]. }\end{array}$ \\
\hline 3. & The signal $\widetilde{\mathbf{h}}_{\mathrm{z}, \mathrm{n}} \in \mathbb{C}^{M_{n g f} L \times 1}$ is random, except where prominent. & $\begin{array}{c}\text { The signal } \widetilde{\mathbf{h}}_{z, \mathrm{n}} \in \mathbb{C}^{M_{n g f} L \times 1} \text { is a structured sparse equivalent CIR } \\
\text { vector. }\end{array}$ \\
\hline 4. & $\mathbf{w}_{z, \mathrm{n}}$ represents arbitrary noise vector & $\begin{array}{c}\mathbf{w}_{\mathrm{z}, \mathrm{n}} \text { represents the Additive White Gaussian Noise of nth } \\
\text { antenna group for zth OFDM symbol }\end{array}$ \\
\hline
\end{tabular}

unrealistic assumption, specifically in wireless communication scenario.

There are two differences between SUCoSaMP and CoSaMP. Firstly, SUCoSaMP estimates the channels; i.e., it recovers the high dimensional sparse vector by utilizing structured sparsity of massive MIMO channels from one vector of low dimension. Secondly, SUCoSaMP adaptively acquires the sparsity level. In contrast, the CoSaMP recovers the sparse vector without exploiting the structured sparsity and it requires prior information of correct sparsity level.

The proposed SUCoSaMP algorithm is exactly the same as CoSaMP algorithm up to step 2.9. The proposed algorithm stops the iteration with fixed sparsity level (i.e., the current value of s) if $\left\|\boldsymbol{v}^{k}\right\|_{2}>\left\|\boldsymbol{v}^{k+1}\right\|_{2}$ and then it performs an additional step, that is, step \# 2.10, to update the sparsity level by adding 1 to the current value of sparsity level (i.e., $s \longleftarrow s+1)$.

There are two different types of iterations in SUCoSaMP, one on $k$ and one on $s$, and finally the iterations on $s$ are stopped if $\left\|\boldsymbol{v}^{k+1}\right\|_{2}>\left\|\boldsymbol{v}_{s-1}\right\|_{2}$. Table 1 elaborates some further details of major steps involved in CoSaMP and SUCoSaMP algorithms. And Table 2 demonstrates the hypothesis of CoSaMP and SUCoSaMP algorithms.
TABLE 3: System parameters.

\begin{tabular}{lc}
\hline Parameter & Type/Value \\
\hline Total number of transmit antennas & 128 \\
Number of transmit antennas in one sub-group & 16 \\
Number of antennas groups $\left(N_{g}\right)$ & 8 \\
Modulation & $16 \mathrm{QAM}$ \\
Guard Interval & 16 \\
Number of pilot subcarriers $\left(N_{p}\right)$ & 32,64 \\
System bandwidth & $20 \mathrm{MHz}$ \\
DFT size & 2048 \\
\hline
\end{tabular}

\section{Simulation Results}

Simulations have been performed in MATLAB in order to verify the effectiveness of the proposed methods. Mean square error performance of proposed scheme is compared with the conventional OMP, CoSaMP, Structured Subspace Pursuit (SSP), and Adaptive Structured Subspace Pursuit (ASSP) algorithms. Simulation parameters are mentioned in Table 3 for the proposed system. The BS has 1D 1x128 antenna array $(M=128)$. The system bandwidth and 


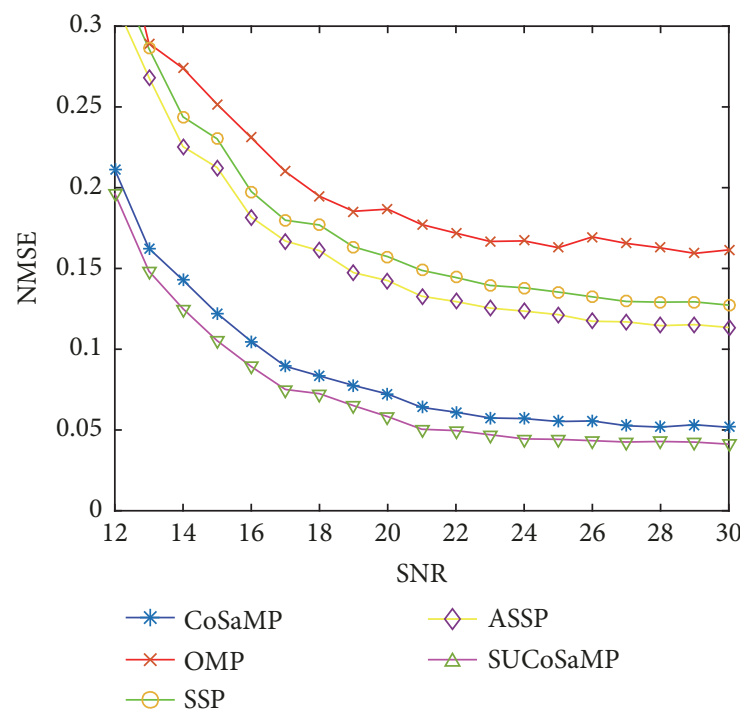

(a)

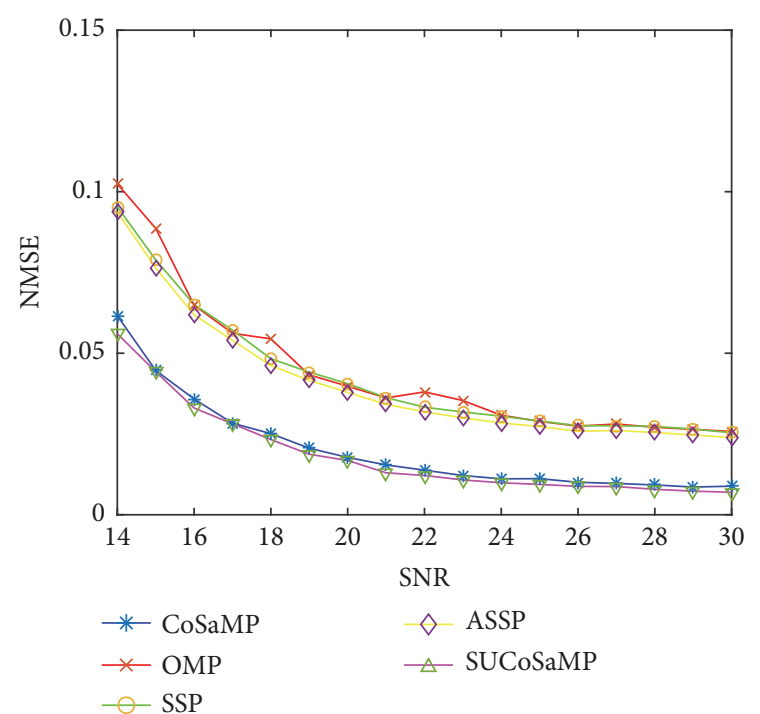

(b)

Figure 5: Comparison of MSE of each algorithm under different SNRs: (a) with $N_{p}=32$, (b) with $N_{p}=64$.

carrier frequency are set to $B=20 \mathrm{MHz}$ and $f_{c}=2 \mathrm{GHz}$, respectively. There are $N_{g}=8$ sub-antenna groups with 16 transmit antennas in each group to ensure the spatial channel sparsity within group. The OFDM subcarriers are set as $N=2048$, guard interval is $N_{G}=16$ which could fight the delay spread up to $6.4 \mu \mathrm{s}$, and 16QAM modulation is used. The numbers of pilot subcarrier $N_{p}$ in OFDM symbol transmitted by each antenna in each antenna group and channel length $L$ are varied over a reasonable range to verify the performance of the proposed system. The pilot positions are uniformly distributed according to (16) and are identical for the entire antennas within one group. The number of multipath channels is randomly chosen and the channel multipath amplitudes and positions follow Rayleigh and random distribution, respectively.

Figure 5 shows the MSE performance of the proposed SUCoSaMP algorithm with different values of $N_{p}$ versus signal to noise ratio (SNR). The performance of SUCoSaMP is compared with the conventional algorithms OMP and CoSaMP and with SSP and ASSP. The sparsity level for OMP, CoSaMP, and SSP is known in simulations whereas the ASSP and proposed SUCoSaMP adaptively acquire the correct sparsity level. It is observed that the channel estimation performance of all the algorithms is improved by increasing pilot subcarriers $N_{p}$. And overall the proposed SUCoSaMP outperforms all the other algorithms. This is because the SUCoSaMP takes full advantage of structured sparsity of massive MIMO channels. Since the prior information of sparsity level of massive MIMO wireless channel is not a realistic assumption, the proposed SUCoSaMP algorithm has a clear advantage over the conventional algorithms. Furthermore the MSE gap between the proposed SUCoSaMP and the conventional algorithm remains almost constant as the SNR increases which makes SUCoSaMP superior in both low and high SNR wireless communication scenarios.
In Figures 6(a)-6(h) individual MSE performance versus SNR of different sub antenna groups (i.e., from antenna group $N_{1}$ to $N_{8}$ ) is presented for $N_{p}=64$ and is compared with conventional algorithms OMP and CoSaMP and with SSP and ASSP. It can be seen in the figures that SUCoSaMP is evidently superior to conventional algorithms. This is because the provided sparsity level for conventional algorithms cannot be the actual sparsity of massive MIMO wireless channels. However, the adaptive process in ASSP and SUCoSaMP is realized by fixed increment in sparsity level; therefore the sparsity level estimation in these algorithms is slightly overestimated or underestimated. It is again observed that with high number of pilot subcarriers $N_{p}$, the performance of SUCoSaMP is improved along with the other algorithms. Furthermore it is observed that the MSE gap between the proposed SUCoSaMP and the conventional algorithm slightly increases or remains almost constant with the increase in SNR resulting in SUCoSaMP being better in different wireless communication environments with respect to SNR.

In Figures 7(a)-7(h) MSE performance versus SNR of individual antenna groups (i.e., from antenna group $N_{1}$ to $N_{8}$ ) is presented with $N_{p}=32$ and comparison is provided with conventional algorithms OMP and CoSaMP and with SSP and ASSP. It can be seen from the figures that the SUCoSaMP algorithm for each antenna group can achieve huge MSE gains over conventional algorithms. However it is observed that the MSE performance of all algorithms slightly degraded with decrease in number of pilot subcarriers $N_{p}$. Results show that SUCoSaMP efficiently considers the effect of SNR and performs better in both high and low SNR scenarios.

\section{Conclusion}

This paper proposes a nonorthogonal pilot design and a CS based algorithm SUCoSaMP to estimate the massive 


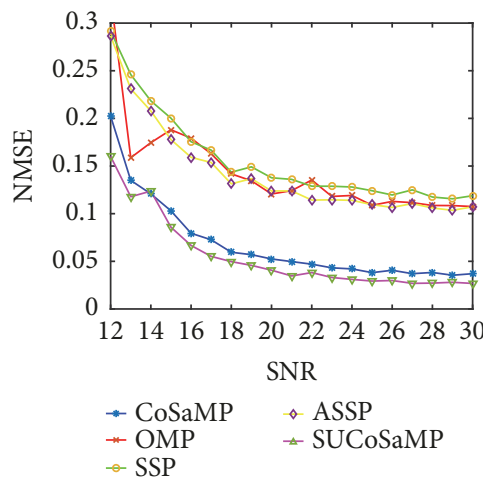

(a)

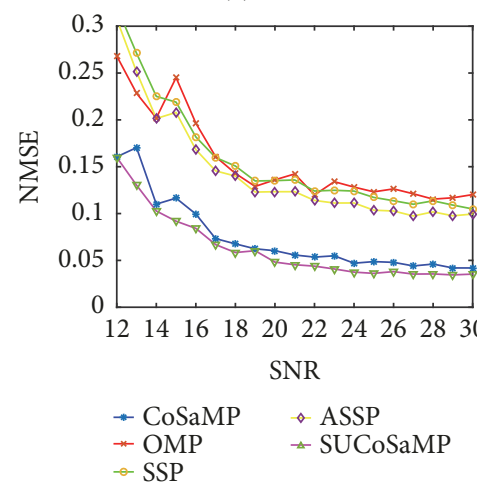

(c)

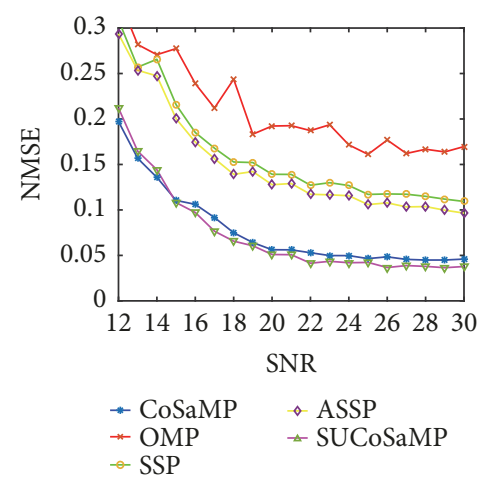

(e)

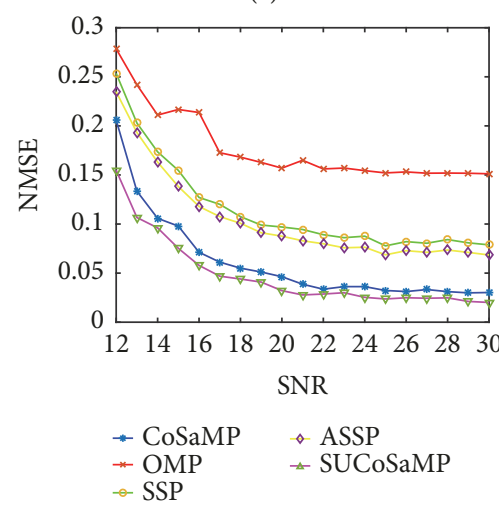

(g)

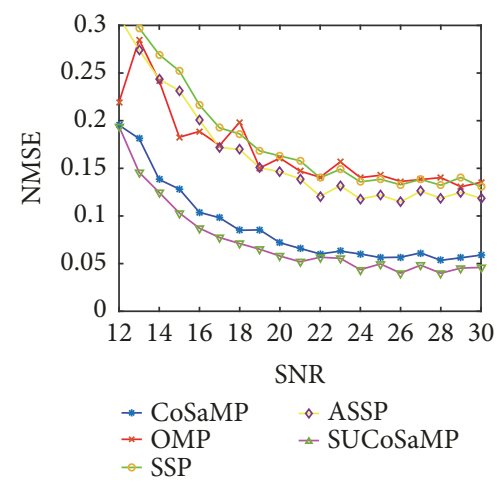

(b)

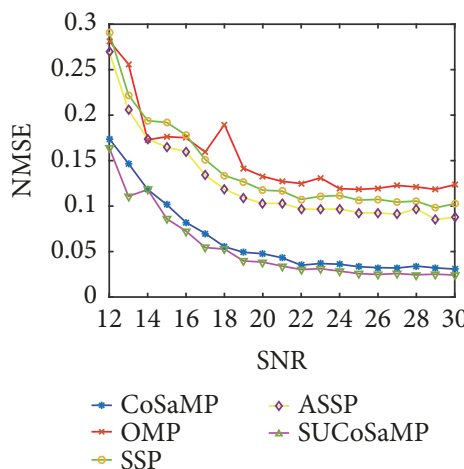

(d)

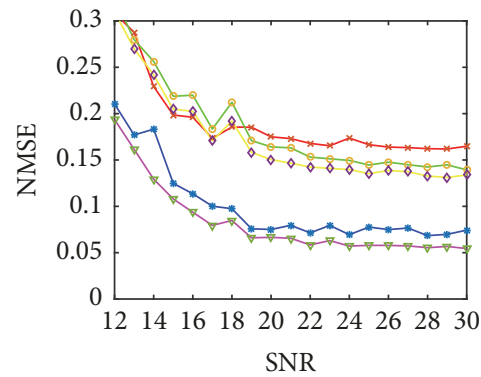

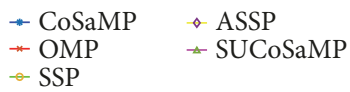

(f)

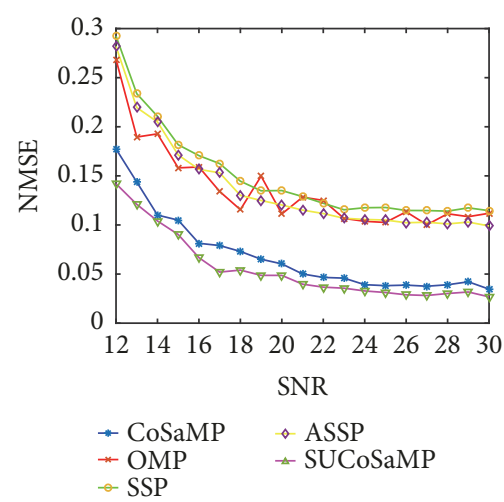

(h)

FIGURE 6: Comparison of MSE of each algorithm under different SNRs for individual antenna groups, with $N_{p}=64$. (a) MSE performance versus SNR of 1st antenna group $\left(N_{1}\right)$. (b) MSE performance versus SNR of 2nd antenna group $\left(N_{2}\right)$. (c) MSE performance versus SNR of 3rd antenna group $\left(N_{3}\right)$. (d) MSE performance versus SNR of 4th antenna group $\left(N_{4}\right)$. (e) MSE performance versus SNR of 5th antenna group $\left(N_{5}\right)$. (f) MSE performance versus SNR of 6th antenna group $\left(N_{6}\right)$. (g) MSE performance versus SNR of 7th antenna group ( $\left.N_{7}\right)$. (h) MSE performance versus SNR of 8th antenna group $\left(N_{8}\right)$. 


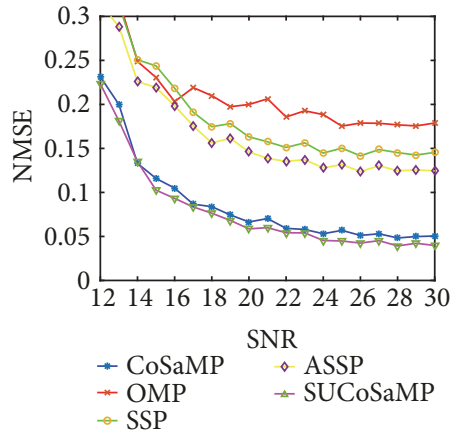

(a)

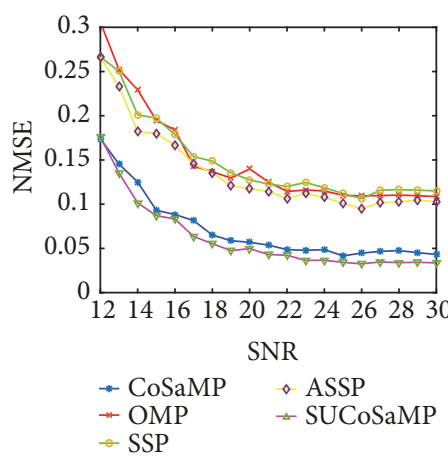

(c)

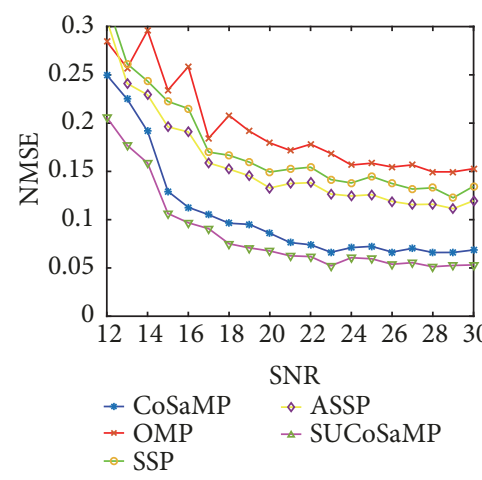

(e)

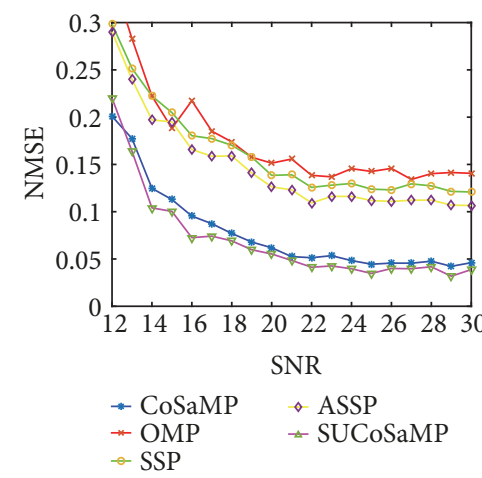

(g)

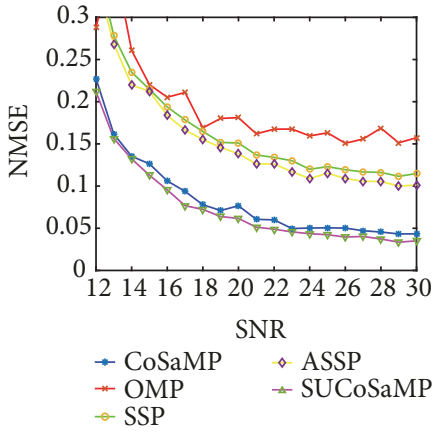

(b)

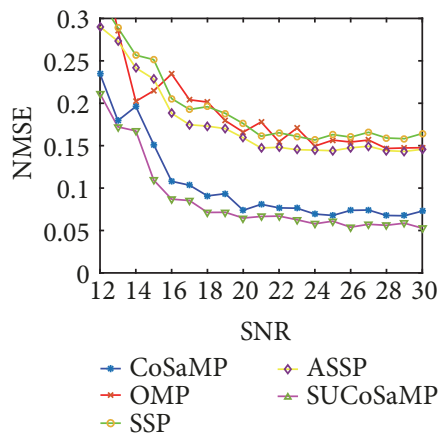

(d)

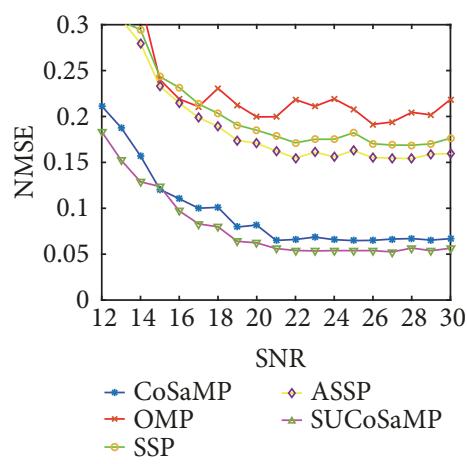

(f)

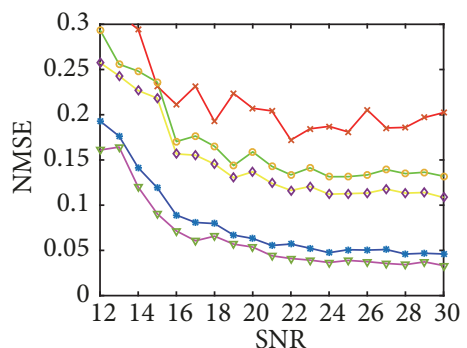

$$
\begin{array}{ll}
\rightarrow \text { CoSaMP } & \rightarrow \text { ASSP } \\
\text { - OMP SSP } & \rightarrow \text { SUCoSaMP }
\end{array}
$$

(h)

FIGURE 7: Comparison of MSE of each algorithm under different SNRs for individual antenna groups, with $N_{p}=32$. (a) MSE performance versus SNR of 1st antenna group $\left(N_{1}\right)$. (b) MSE performance versus SNR of 2nd antenna group $\left(N_{2}\right)$. (c) MSE performance versus SNR of 3rd antenna group $\left(N_{3}\right)$. (d) MSE performance versus SNR of 4th antenna group $\left(N_{4}\right)$. (e) MSE performance versus SNR of 5th antenna group $\left(N_{5}\right)$. (f) MSE performance versus SNR of 6th antenna group $\left(N_{6}\right)$. (g) MSE performance versus SNR of 7th antenna group ( $\left.N_{7}\right)$. (h) MSE performance versus SNR of 8th antenna group $\left(N_{8}\right)$. 
MIMO channels. The reduction of high pilot overhead in massive MIMO systems and the recovery ability when the sparsity level of massive MIMO channels is unknown are the main focus of this research. By taking advantage of spatial and temporal common sparsity of massive MIMO channels in delay domain, the proposed nonorthogonal pilot design and channel estimation scheme under the frame work of CS theory significantly reduce the pilot overheads for massive MIMO systems and also outperform the conventional algorithms in performance. This research may be extended by incorporating the proposed schemes in the multicell scenarios.

\section{Data Availability}

The data used to support the findings of this study are included within the article.

\section{Conflicts of Interest}

The authors declare that there are no conflicts of interest regarding the publication of this paper.

\section{References}

[1] C.-X. Wang, F. Haider, X. Gao et al., "Cellular architecture and key technologies for $5 \mathrm{G}$ wireless communication networks," IEEE Communications Magazine, vol. 52, no. 2, pp. 122-130, 2014.

[2] F. Rusek, D. Persson, and B. K. Lau, "Scaling up MIMO: opportunities and challenges with very large arrays," IEEE Signal, 2013.

[3] H. Yang, Y. Fan, D. Liu, and Z. Zheng, "Compressive sensing and prior support based adaptive channel estimation in massive MIMO," in Proceedings of the 2nd IEEE International Conference on Computer and Communications, ICCC '16, IEEE, 2016.

[4] J. G. Andrews, S. Buzzi, and W. Choi, "What will 5G be?" IEEE Journal on Selected Areas in Communications, vol. 32, no. 6, pp. 1065-1082, 2014.

[5] W. H. Chin, Z. Fan, and R. Haines, "Emerging technologies and research challenges for 5G wireless networks," IEEE Wireless Communications Magazine, vol. 21, no. 2, pp. 106-112, 2014.

[6] W. Xu, T. Shen, Y. Tian, and Y. Wang, "Compressive channel estimation exploiting block sparsity in multi-user massive MIMO systems," in Proceedings of the 2017 IEEE Wireless Communications and Networking Conference, WCNC '17, IEEE, 2017.

[7] J. Zhang, B. Zhang, S. Chen, and X. Mu, "Pilot contamination elimination for large-scale multiple-antenna aided OFDM systems," IEEE Journal, 2014.

[8] E. Bjonson, J. Hoydis, and M. Kountouris, "Massive MIMO systems with non-ideal hardware: energy efficiency, estimation, and capacity limits," IEEE Transactions, 2014.

[9] Y. S. Cho, J. Kim, W. Yang, and C. Kang, MIMO-OFDM Wireless Communications with MATLAB, 2010.

[10] Y. Xu, G. Yue, and S. Mao, "User grouping for massive MIMO in FDD systems: new design methods and analysis," IEEE Access, vol. 2, pp. 947-959, 2013.
[11] B. Hassibi and B. M. Hochwald, "How much training is needed in multiple-antenna wireless links?" IEEE Transactions on Information Theory, vol. 49, no. 4, pp. 951-963, 2003.

[12] L. Correia, Mobile Broadband Multimedia Networks: Techniques, Models and Tools for 4G, 2010.

[13] Z. Zhou, X. Chen, and D. Guo, "Sparse channel estimation for massive MIMO with 1-bit feedback per dimension," in Proceedings of the IEEE Wireless Communications and Networking Conference, WCNC '17, 2017.

[14] E. Bjornson and B. Ottersten, "A framework for training-based estimation in arbitrarily correlated Rician MIMO channels with Rician disturbance," IEEE Transactions on Signal Processing, 2010.

[15] X. Ma, F. Yang, S. Liu, and J. Song, “Training sequence design and optimization for structured compressive sensing based channel estimation in massive MIMO systems," in Proceedings of the Globecom Work (GC '16), 2016.

[16] I. Barhumi, G. Leus, and M. Moonen, "Optimal training design for MIMO OFDM systems in mobile wireless channels," IEEE Transactions on Signal Processing, vol. 51, no. 6, pp. 1615-1624, 2003.

[17] H. Minn and N. Al-Dhahir, "Optimal training signals for MIMO OFDM channel estimation," IEEE Transactions on Wireless Communications, 2006.

[18] Y.-H. Nam, Y. Akimoto, Y. Kim, M.-I. Lee, K. Bhattad, and A. Ekpenyong, "Evolution of reference signals for LTE-advanced systems," IEEE Communications Magazine, vol. 50, no. 2, pp. 132-138, 2012.

[19] L. Lu, G. Y. Li, and A. L. Swindlehurst, "An overview of massive MIMO: benefits and challenges," IEEE Journal, 2014.

[20] N. Gonzalez-Prelcic, K. T. Truongt, C. Rusu, and R. W. Heath, "Compressive channel estimation in FDD multi-cell massive MIMO systems with arbitrary arrays," in Proceedings of the 2016 IEEE Globecom Workshops, GC Wkshps '16, 2016.

[21] L. Dai, Z. Wang, and Z. Yang, "Spectrally efficient timefrequency training OFDM for mobile large-scale MIMO systems," IEEE Journal on Selected Areas in Communications, vol. 31, no. 2, pp. 251-263, 2013.

[22] M. S. Sim, J. Park, and C.-B. Chae, "Compressed channel feedback for correlated massive MIMO," Commun., 2016.

[23] Z. Gao, L. Dai, and Z. Wang, "Structured compressive sensing based superimposed pilot design in downlink large-scale MIMO systems," IEEE Electronics Letters, vol. 50, no. 12, pp. 896-898, 2014.

[24] C. Qi and L. Wu, "Uplink channel estimation for massive MIMO systems exploring joint channel sparsity," IEEE Electronics Letters, vol. 50, no. 23, pp. 1770-1772, 2014.

[25] Z. Gao, L. Dai, Z. Lu, and C. Yuen, "Super-resolution sparse MIMO-OFDM channel estimation based on spatial and temporal correlations," IEEE Communications Letters, vol. 18, no. 7, pp. 1266-1269, 2014.

[26] S. L. H. Nguyen and A. Ghrayeb, "Compressive sensing-based channel estimation for massive multiuser MIMO systems," in Proceedings of the 2013 IEEE Wireless Communications and Networking Conference, WCNC '13, 2013.

[27] Z. Gao, L. Dai, Z. Wang, and S. Chen, "Spatially common sparsity based adaptive channel estimation and feedback for FDD massive MIMO," IEEE Transactions on Signal Processing, vol. 63, no. 23, pp. 6169-6183, 2015.

[28] W. Shen, L. Dai, B. Shim, and S. Mumtaz, "Joint CSIT acquisition based on low-rank matrix completion for FDD massive 
MIMO systems," IEEE Communications Letters, vol. 19, no. 12, pp. 2178-2181, 2015.

[29] X. Ma, F. Yang, S. Liu, and J. Song, "Doubly selective channel estimation for MIMO systems based on structured compressive sensing," in Proceedings of the 2017 13th International Wireless Communications and Mobile Computing Conference (IWCMC '17), 2017.

[30] Y. Ding and B. D. Rao, "Dictionary learning based sparse channel representation and estimation for FDD massive MIMO systems," 2016.

[31] B. Gong, L. Gui, Q. Qin, and X. Ren, "Block distributed compressive sensing-based doubly selective channel estimation and pilot design for large-scale MIMO systems," IEEE Transactions on Vehicular Technology, vol. 66, no. 10, pp. 9149-9161, 2017.

[32] S. Sun and T. S. Rappaport, "Millimeter Wave MIMO channel estimation based on adaptive compressed sensing," in Proceedings of the 2017 IEEE International Conference on Communications Workshops (ICC Workshops '17), 2017.

[33] J. Choi, D. J. Love, and P. Bidigare, "Downlink training techniques for FDD massive MIMO systems: Open-loop and closed-loop training with memory," IEEE Journal of Selected Topics in Signal Processing, vol. 8, no. 5, pp. 802-814, 2014.

[34] D. Hu, X. Wang, and L. He, "A new sparse channel estimation and tracking method for time-varying OFDM systems," IEEE Transactions on Vehicular Technology, vol. 62, no. 9, pp. 46484653, 2013.

[35] T. Santos, J. Karedal, and P. Almers, "Modeling the ultrawideband outdoor channel: measurements and parameter extraction method," IEEE Transactions on Wireless Communications, vol. 9, no. 1, pp. 282-290, 2010.

[36] I. E. Telatar and D. N. Tse, "Capacity and mutual information of wideband multipath fading channels," Institute of Electrical and Electronics Engineers Transactions on Information Theory, vol. 46, no. 4, pp. 1384-1400, 2000.

[37] I. Khan, M. Singh, and D. Singh, "Compressive sensing-based sparsity adaptive channel estimation for 5G massive MIMO systems," Applied Sciences, vol. 8, no. 5, p. 754, 2018.

[38] Y. Nan, L. Zhang, and X. Sun, "Weighted compressive sensing based uplink channel estimation for TDD massive MIMO sytems," IET Communications, vol. 11, no. 3, pp. 355-361, 2017.

[39] Z. Gao, L. Dai, W. Dai, B. Shim, and Z. Wang, "Structured compressive sensing-based spatio-temporal joint channel estimation for FDD massive MIMO," IEEE Transactions on Communications, vol. 64, no. 2, pp. 601-617, 2016.

[40] J. Choi, B. Shim, Y. Ding, B. Rao, and D. Kim, "Compressed sensing for wireless communications: useful tips and tricks," IEEE Communications Survey and Tutorials, vol. 19, no. 3, pp. 1527-1550, 2017.

[41] A. Lozano and N. Jindal, "Optimum pilot overhead in wireless communication: a unified treatment of continuous and blockfading channels," in Proceedings of the 2010 European Wireless Conference (EW'10), 2010. 


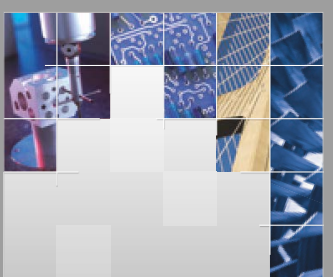

\section{Enfincering}
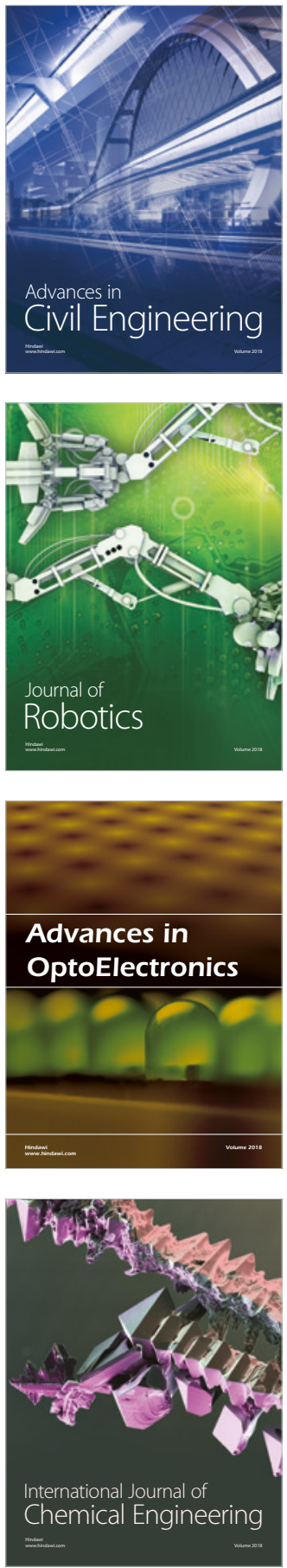

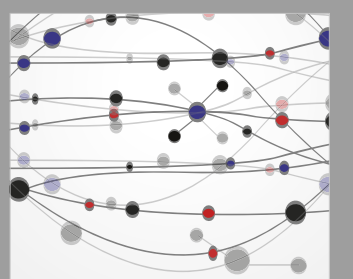

\section{Rotating \\ Machinery}

The Scientific World Journal

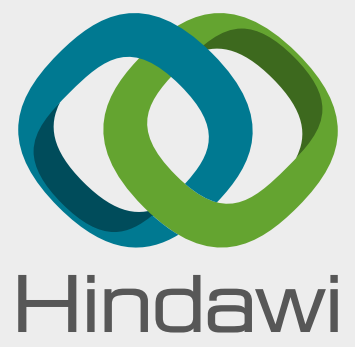

Submit your manuscripts at

www.hindawi.com
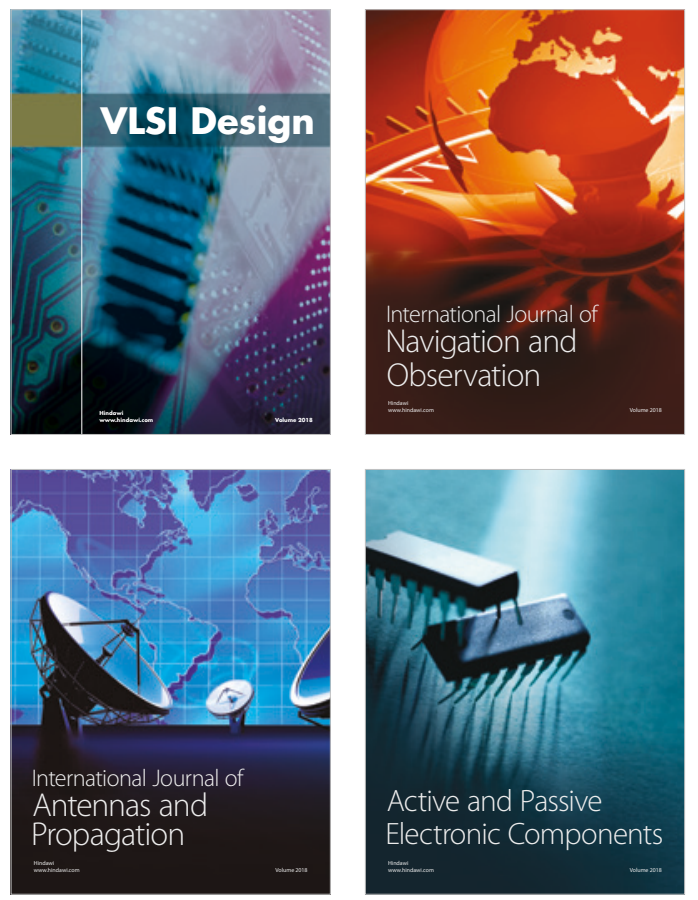
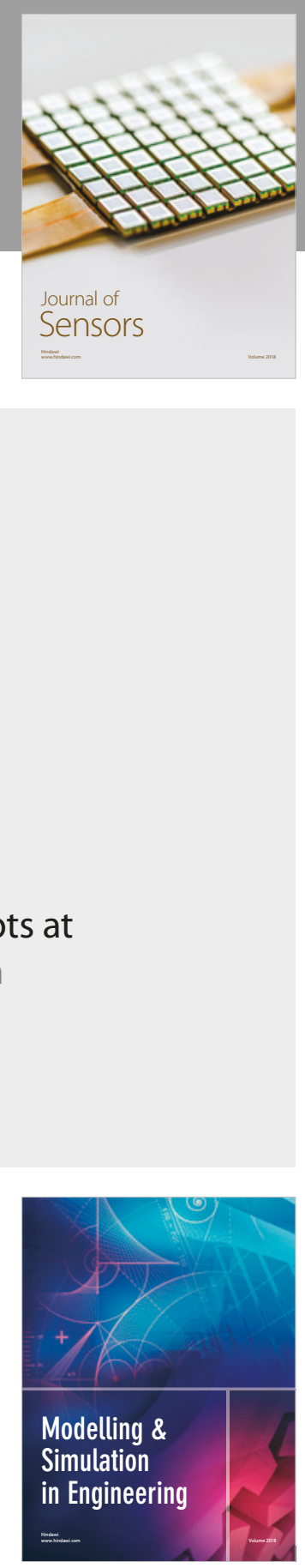

\section{Advances \\ Multimedia}
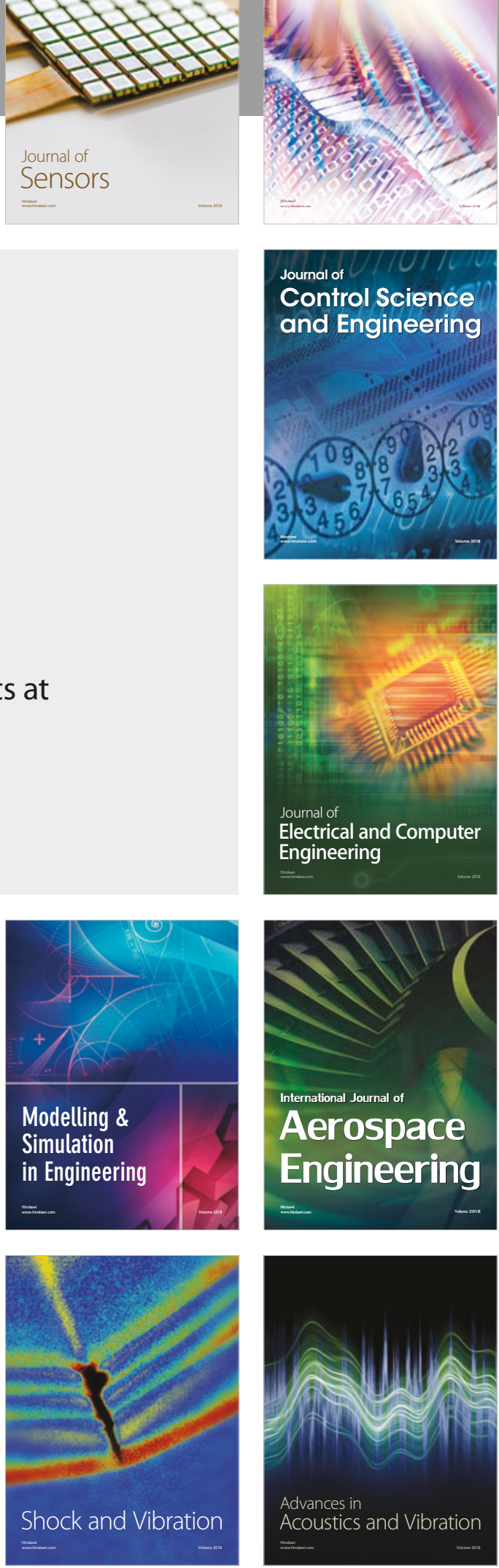